\title{
August 2013 Pulmonary Case of the Month: Aids for Diagnosis
}

\author{
Lewis J. Wesselius, MD \\ Department of Pulmonary Medicine \\ Mayo Clinic Arizona \\ Scottsdale, AZ
}

\section{History of Present IIIness}

An 80 year old man was referred for evaluation of cough, weakness and weight loss over 2-3 months. He had a chest radiograph 6 weeks ago showing a right lower lobe infiltrate. He was treated with levofloxacin and prednisone without improvement.

\section{$P M H, S H, F H$}

He had a history of hypertension, type 2 diabetes mellitus, hyperlipidemia, and hypothyroidism.

He was born in China, had lived in Philippines, Hong Kong and Phoenix, the later for the last 23 years. He was lifetime nonsmoker and rarely used ethanol. He had no pets, unusual exposures, and no known tuberculosis exposure (last skin test was negative 10 years ago).

His father died at age 79 from coronary artery disease. His mother had "intestinal cancer". He has a sister with diabetes mellitus.

\section{Medications}

- Atorvastatin $10 \mathrm{mg} / \mathrm{day}$

- Doxazosin $2 \mathrm{mg} / \mathrm{day}$

- Levothyroxin $50 \mathrm{mcg} /$ day

- Metformin $500 \mathrm{mg}$ bid

- Metoprolol XL 25 mg/day

- Zantac $50 \mathrm{mg}$ bid

- Recent Levaquin/Prednisone

\section{Physical Examination}

Blood pressure 130/62, Pulse 72, afebrile, SpO2 97\%, body mass index 19.5 Chest: lungs were clear to auscultation and percussion.

There were no significant findings on physical examination. 


\section{Laboratory}

Laboratory evaluation revealed a slight anemia with hemoglobin of $12.6 \mathrm{~g} / \mathrm{dL}$ but a normal white count of $7.9 \times 10^{6}$ cells $/ \mathrm{mcL}$ with $0.06 \%$ eosinophils. Erythrocyte sedimentation rate was $53 \mathrm{~mm} / \mathrm{hr}$. Albumin was slightly low at $2.9 \mathrm{gm} / \mathrm{dL}$.

\section{Chest Radiography}

His chest $x$-ray is shown in figure 1.
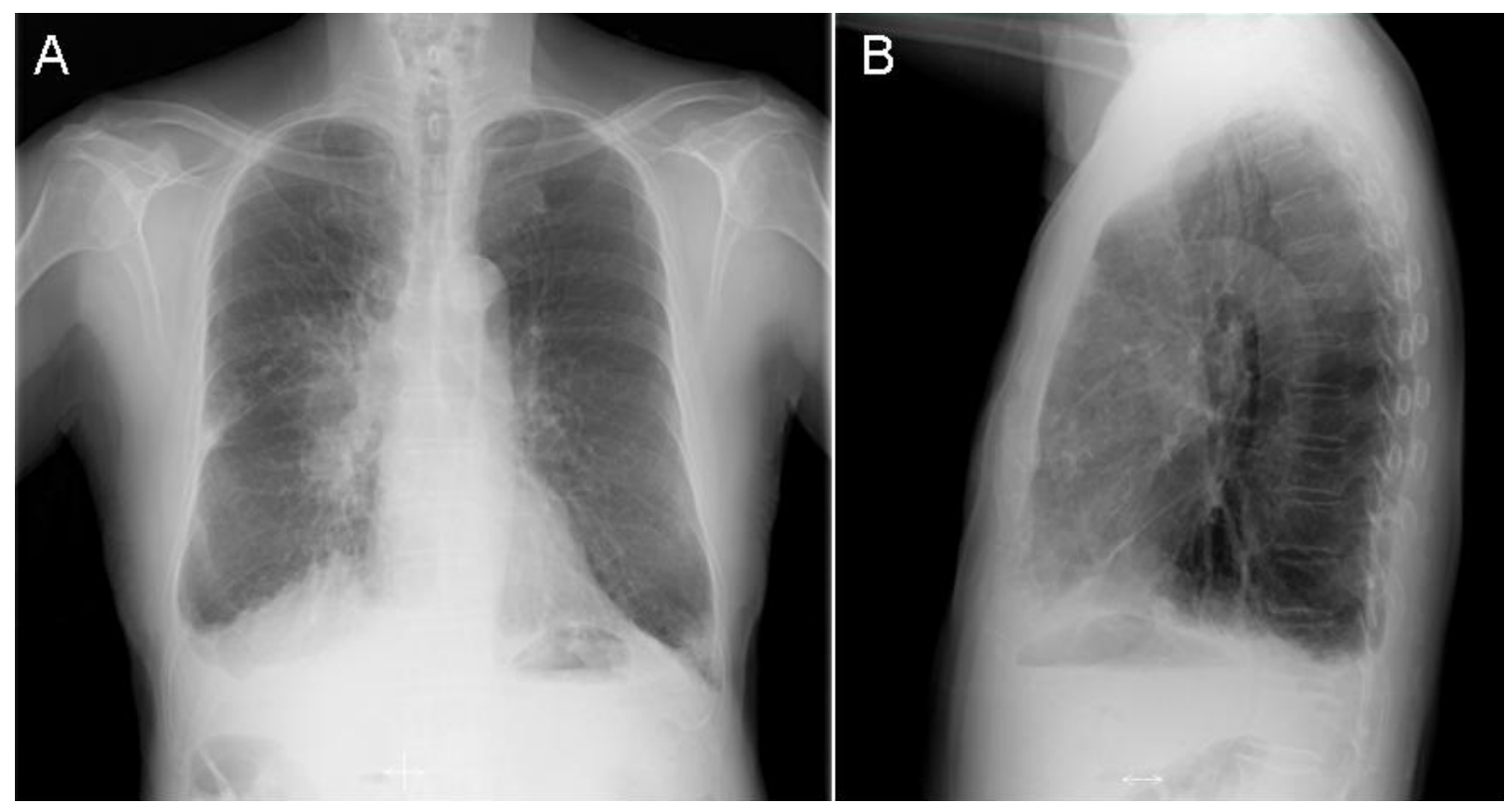

Figure 1. Patient's posterior-anterior chest radiograph (Panel A) and lateral (Panel B).

Which of the following best describes the chest x-ray?

1. Multifocal nodular consolidation

2. Left lower lobe collapse

3. Right hilar fullness

4. 1 and 3

5. All of the above 


\section{Correct!}

\section{1 and 3}

The chest radiograph shows several areas of what appears to be nodular consolidation (Figure 2). In addition, there is a right hilar fullness best visualized on the PA chest radiograph. There is no evidence of left lower lobe collapse which would be indicated by volume loss and a sail-like density often seen on the PA view behind the heart shadow.

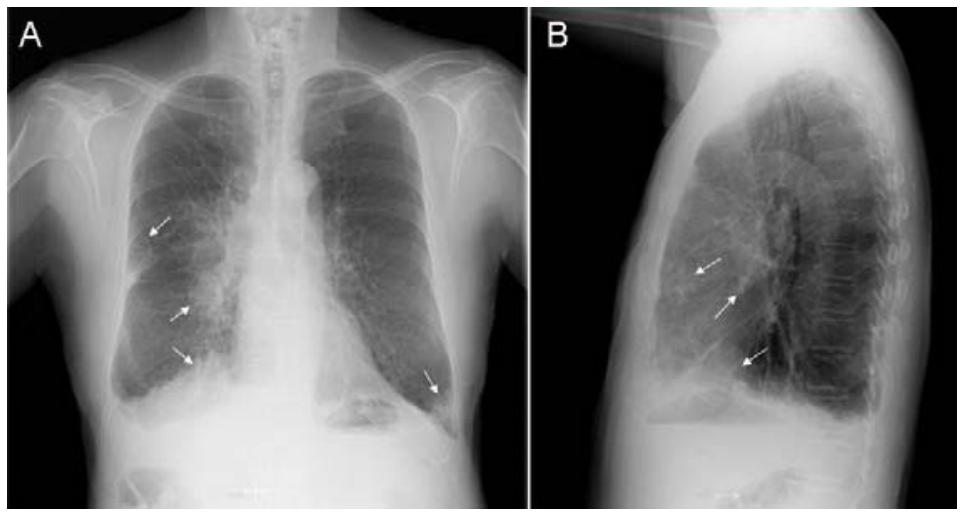

Figure 2. Patient's chest x-ray showing areas of nodular consolidation (arrows) and right hilar fullness.

A CT scan of the chest was performed to better define the abnormalities seen on chest x-ray (Figure 3).
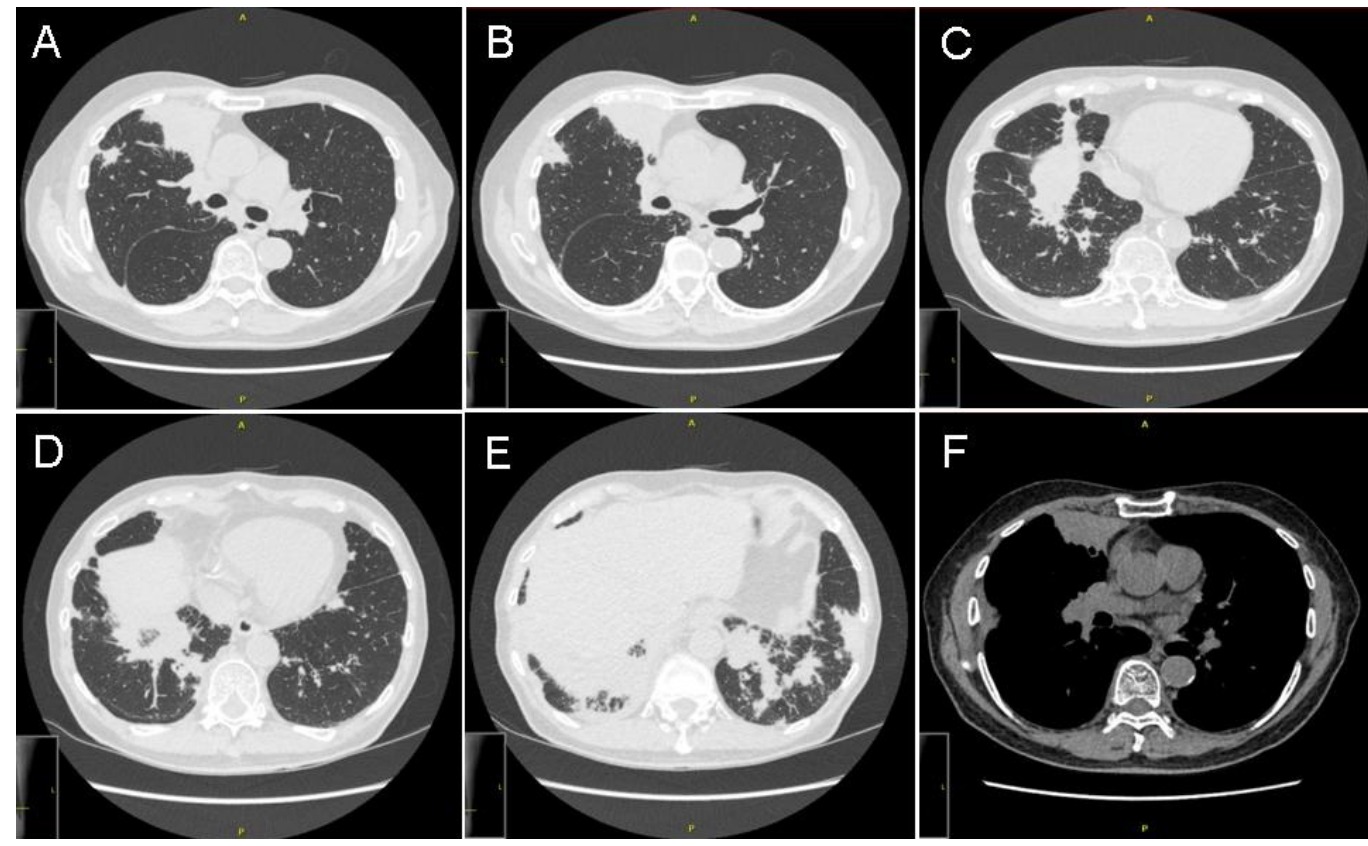

Figure 3. Selected thoracic CT lung window images (Panels A-E) and a soft tissue window through the right hilum (Panel F). 
Which of the following is the best next step in evaluating the patient?

1. ${ }^{18 F}$ FDG-PET scanning

2. Bronchoscopy with bronchoalveolar lavage

3. Careful observation with serial chest $\mathrm{x}$-rays

4. Needle biopsy of the lung

5. VATS lung biopsy 


\section{Correct! \\ 2. Bronchoscopy with bronchoalveolar lavage}

The patient has an undiagnosed diffuse lung process. Bronchoscopy with bronchoalveolar lavage would probably have the best risk-benefit ratio. The patient has had an ongoing process for several weeks and observation in hopes that the process will resolve seems unlikely. Needle aspiration and videoassisted thorascopic (VATS) lung biopsy may also reveal a diagnosis but are more invasive and have higher complication rates than bronchoscopy. ${ }^{18 F}$ FDGPET scanning is most useful for staging lung cancer.

Bronchoscopy revealed no bronchial abnormalities and the bronchoalveolar lavage stains and smears were negative. However, the cultures grew Cryptococcus neoformans.

Which of the following is true regarding a positive culture for Cryptococcus from the bronchoalveolar lavage fluid?

1. Cryptococcus pneumonia is usually seen in immunocompromised hosts

2. Cryptococcus is a frequent contaminant of bronchoalveolar lavage fluid

3. Cryptococcus should prompt an investigation for possible meningitis/encephalitis

4. 1 and 3

5. All of the above 


\section{Correct!}

\section{1 and 3}

Cryptococcosis is a potentially fatal fungal disease (1). It is caused by one of two species; Cryptococcus neoformans or Cryptococcus gattii. Cryptococcosis is believed to be acquired by inhalation and is frequently seen in immunocompromised hosts and is a defining opportunistic infection for AIDS. Other conditions which pose an increased risk for cryptococcosis include lymphomas, sarcoidosis, liver cirrhosis and patients on long-term corticosteroid therapy. It has only rarely been reported in immunocompetent hosts or as a nonpathogenic colonizing organism. Pulmonary cryptococcosis is frequently associated with meningitis/encephalitis especially in immunocompromised hosts. The patient's cerebrospinal fluid cryptococcal antigen was negative. However, the CSF had a slightly elevated cell count of 7 cells/HPF all lymphocytes. The protein was also slightly elevated at $61 \mathrm{mg} / \mathrm{dL}$ and his glucose was slightly low at $53 \mathrm{mg} / \mathrm{dL}$. An infectious disease consultant interpreted these findings as consistent with cryptococcal meningitis.

Which of the following should be ordered for the work up of this possibly immunocompromised patient?

1. CD4 lymphocyte count

2. HIV

3. Total immunoglobulins and IgG

4. 1 and 3

5. All of the above 


\section{Correct! \\ 5. All of the above}

The most frequent cause of immunocompromise in a patient with cryptococcosis is AIDS. Our patient's HIV was negative as was his anti-HTLV I/II. Crytococcosis has been reported in patients with hypogammaglobulinemia but the patient's immunoglobulins were normal. However, his CD4 count was low at 154 cells $/ \mathrm{mm}^{3}$. Our infectious disease consultant thought this likely represented idiopathic CD4 lymphocytopenia (ICL).

$I C L$ is a rare disorder with a poorly defined incidence (2). There is no gender bias and the mean age in one review was 43 years. There is no evidence of any transmissible agent as a cause but the underlying mechanism is unknown. The most common clinical presentation is opportunistic infections particularly with Cryptococcus or atypical Mycobacteria sp. Pneumocystis, human papilloma virus, and shingles have also been reported to be associated with ICL. The CDC has defined ICL by 3 criteria:

1. Absolute CD4 T-lymphocytes $<300$ on two occasions 6 weeks apart

2. HIV negative.

3. Absence of any defined immunodeficiency or therapy associated with reduced CD4 counts.

Cryptococcal meningitis can be particularly difficult to diagnose in this population (3) and was the reason for the diagnosis of cryptococcal meningitis with the minimal CSF findings.

The patient was being treated with fluconazole at last report and stable. There is no specific treatment for ICL although the National Institutes of Health has an ongoing clinical trial with IL-7 treatment. Prophylaxis against Pneumocystis is recommended with CD4 $<200$ cells $/ \mathrm{mm}^{3}$.

\section{References}

1. Smith JA, Kauffman CA. Pulmonary fungal infections. Respirology. 2012;17(6):913-26.[CrossRef] [PubMed]

2. Luo L and Li T, Idiopathic CD4 lymphocytopenia and opportunistic infections - an update. FEMS Immunol Med Microbiol. 2008;54:283-9. [CrossRef] [PubMed]

3. Sancesario G, Palmieri G, Viola G, Fontana C, Perfetti A, Anemona L, Floris R, Marziali S, Bernardi G, Spagnoli LG. Difficultry diagnosing chronic cryptococcal meningitis in idiopathic CD4 lymphocytopenia. Neurol Sci 2011:32(3):519-24. [CrossRef] [PubMed] 